\title{
Mini Core Collection as a Resource to Identify New Sources of Variation
}

\author{
H.D. Upadhyaya,^ N. Dronavalli, S.L. Dwivedi, J. Kashiwagi, L. Krishnamurthy, \\ S. Pande, H.C. Sharma, V. Vadez, S. Singh, R.K. Varshney, and C.L.L. Gowda
}

\begin{abstract}
In chickpea, bottlenecks associated with its domestication and low use of germplasm in improvement programs have resulted in a narrow genetic base and its vulnerability to abiotic and biotic stresses. The core and mini core collections, representing diversity in the entire collection, have been advocated for enhanced utilization of germplasm in crop improvement. A chickpea mini core (211 accessions) was evaluated for agronomic traits from 2000 and 2001 to 2003 and 2004 in post-rainy seasons under irrigated and non-irrigated conditions. The published information on the response of chickpea mini core accessions to stress revealed that 40 accessions had resistance to abiotic stress, 31 to biotic stress, and 24 had no resistance to either of the stresses. The abiotic and biotic stress resistant groups had six accessions in common. The mini core collection accessions were also a part of composite collection accessions in chickpea, which was genotyped using 48 simple sequence repeats (SSRs; BMC Plant Biol. 8:106, 2008). The agronomic evaluation, stress response, and molecular profiling data on 93 accessions, including four controls, were used to identify genetically diverse germplasm with agronomically beneficial traits. A number of genetically diverse accessions possessing agronomically beneficial traits, such as ICC 440 , 637, 1098, 3325, 3362, 4872, 7441, 8621, 9586, $10399,12307,14402,15680$, and 15686, which meet breeders' needs, have been identified for use in breeding and genetics to map genomic regions associated with beneficial traits and as source materials for developing high yielding and widely adapted chickpea cultivars with multiple resistance to abiotic and biotic stress.
\end{abstract}

H.D. Upadhyaya, N. Dronavalli, S.L. Dwivedi, J. Kashiwagi, L. Krishnamurthy, S. Pande, H.C. Sharma, V. Vadez, S. Singh, R.K. Varshney, and C.L.L. Gowda, Genetic Resources, CGIAR Grain Legumes Research Program, International Crops Research Institute for the SemiArid Tropics (ICRISAT), Patancheru, AP, 502 324, India. Received 23 Apr. 2013. *Corresponding author (h.upadhyaya@cgiar.org).

Abbreviations: $\mathrm{AB}$, ascochyta blight; $\mathrm{BGM}$, botrytis gray mold; FW, fusarium wilt; LPB, legume pod borer; RD, root depth; REML, residual (or restricted) maximum likelihood; SSR, simple sequence repeat.

C HICKPEA (Cicer arietinum L.) is the second most important legume after beans, with a total production of 11.6 million tons $(\mathrm{Mt})$ from $13.2 \mathrm{~m}$ ha. However, it ranks fifth in productivity after faba bean, pea, lupin, and lentil. India accounts for $70.7 \%$ of the world chickpea production followed by Australia (4.4\%), Pakistan (4.3\%), Turkey (4.2\%), Myanmar (4.0\%), Ethiopia (2.8\%), Iran (2.5\%), USA (0.84\%), Canada (0.78\%), and Mexico (0.62\%) (FAO, 2011). Chickpea seeds are rich in protein, starch, fiber, minerals, and vitamins, which make it one of the best nutritionally balanced pulses for human consumption (Jukanti et al., 2012). However, like any other pulses, the chickpea seeds also contain antinutritional factors, which can be reduced or eliminated by cooking. Chickpea fixes atmospheric nitrogen up to $141 \mathrm{~kg} \mathrm{ha}^{-1}$ (Rupela, 1987), which helps reduce the input cost for the current and the succeeding crop. The two distinct forms of cultivated chickpeas are desi (small seeds, angular ram's head shape, and colored seeds with high percentage of fiber) and kabuli (large-seeds, irregular rounded, owl's-head shape, and beige colored seeds with a low percentage of fiber) types. An intermediate form recognized as a pea-shaped type also exists and is characterized by medium

Published in Crop Sci. 53:2506-2517 (2013).

doi: 10.2135/cropsci2013.04.0259

(C) Crop Science Society of America | 5585 Guilford Rd., Madison, WI 53711 USA

All rights reserved. No part of this periodical may be reproduced or transmitted in any form or by any means, electronic or mechanical, including photocopying, recording, or any information storage and retrieval system, without permission in writing from the publisher. Permission for printing and for reprinting the material contained herein has been obtained by the publisher. 
to small seed size and cream colored seeds. Both desi and kabuli are easily hybridized, but there are strong consumer and culinary preferences for desi or kabuli chickpeas.

Chickpea is grown as a winter crop in the Indian subcontinent (October-November to March-April) on receding soil moisture, mostly on marginal soils. The main reason for low productivity in chickpea is the adverse ecologies in which it is cropped and its vulnerability to abiotic and biotic stress (Dwivedi et al., 2005, and references therein). Drought, heat, and salinity among the abiotic stresses and fusarium wilt (Fusarium oxysporum f. sp. ciceri), dry root rot (Rhizoctinia bataticola), ascochyta blight (Ascochyta rabiei; AB), botrytis gray mold (Botrytis cinerea), pod borer (Helicoverpa armigera), and leaf miner (Liriomyza cicerina) among the biotic stresses are the major yield reducing constraints, which together cause annual yield loss of US $\$ 4.4$ billion (Ryan, 1997). About one third of these losses can be recovered through genetic enhancement of yield potential by augmenting the productivity genes and resistance to biotic and abiotic stress.

The cultivated chickpea has a narrow genetic base (Kumar et al., 2004) mainly because of the breeder's reluctance to introduce exotic germplasm (wild species, landraces, and exotic lines) due to the linkage drag often associated with the use of such germplasm in breeding programs. Thus, breeders tend to concentrate on adapted and improved materials and avoid wild species, landraces, and exotic lines available in genebanks (Nass and Paterniani, 2000), thereby widening the gap between available genetic resources and their use in breeding programs (Marshall, 1989). Large diversity among chosen parental lines is essential for the success of any recombinant breeding program, specifically when the traits under improvement are quantitative, highly variable, and show high genotype $\times$ environment interactions. Plant architecture and agronomic traits including yield and yield components, and response to crop husbandry are the most important agronomic traits and contribute to the yield potential of a genotype. Identification of source lines for different traits and utilizing them for recombinant breeding is critical for developing chickpea cultivars to meet the emerging challenges to agricultural production.

Availability of trait-specific germplasm and its judicious use is critical for the success of any breeding program. Reduced subsets of germplasm, such as core (Frankel, 1984) or mini core (Upadhyaya and Ortiz, 2001) collections, are a cost effective means of identifying accessions with desirable agronomic and nutritional quality traits and resistance or tolerance to biotic and abiotic stress. The chickpea mini core, which consists of 211 accessions and represent both geographical and biological diversity present in cultivated chickpea germplasm (Upadhyaya and Ortiz, 2001), is an ideal resource for multilocation evaluations to identifying new sources of variation for use in chickpea breeding and genomics. The aims of the present investigation were to (i) assess agronomic performance of the chickpea mini core accessions, and (ii) identify traitspecific genetically diverse germplasm for use in breeding programs. We evaluated the chickpea mini core collection (Upadhyaya and Ortiz, 2001) accessions in multienvironment trials to assess the agronomic performance, while the previously published information on molecular profiling (Upadhyaya et al., 2008) and response to abiotic (Serraj et al., 2004; Kashiwagi et al., 2005, 2006b, 2008, 2010; Vadez et al., 2007; Parameshwarappa and Salimath, 2008; Mulwa et al., 2010; Krishnamurthy et al., 2010; 2011a, 2011b; Upadhyaya et al., 2011; Zaman-Allah et al., 2011a, 2011b) and biotic stress (Pande et al., 2006; ICRISAT, 2009; Mulwa et al., 2010; Taran et al., 2010) was superimposed on agronomic data generated in the present study to identify genetically diverse accessions with beneficial agronomic traits.

\section{MATERIALS AND METHODS}

The experimental materials consisted of 211 chickpea mini core accessions and four controls (Annigeri, G 130, L 550, and ICCV 2). Annigeri (ICC 4918) is an early-maturing desi cultivar adapted in peninsular India, while G 130 (ICC 4948), another desi cultivar but with late maturity, is adapted in northern India. L 550 (ICC 4973) is a high-yielding, mediumduration kabuli cultivar, tolerant to root knot nematode, but susceptible to fusarium wilt and ascochyta blight, and adapted to irrigated conditions in India (Dua et al., 2001). ICCV 2 is an early-maturing and fusarium wilt-resistant kabuli cultivar, adapted in peninsular India (Kumar et al., 1985).

The experiments were conducted in 7 Vertisols (Kasireddipally series-isohypothermic Typic Pellustert) (El-Swaify et al., 1985) environments during 2000 and 2001 to 2003 and 2004 post-rainy seasons (October-November to February-March) under irrigated (one irrigation at flowering and another at grain filling stage, each time plots receiving $50 \mathrm{~mm}$ water) and nonirrigated (residual moisture after cessation of rains) conditions at Patancheru, India. The weather data revealed that the 2000 to 2001 and 2001 to 2002 crop seasons had similarities in most of the parameters, while the 2003 to 2004 crop season differed in terms of maximum and minimum temperatures, relative humidity, solar radiation, and bright sunshine hours (Table 1). The crop in 2000 to 2001 and 2001 to 2002 seasons received 20 to $22 \mathrm{~mm}$ of rain water during the late grain filling stage, while the 2002 to 2003 crop received $55 \mathrm{~mm}$ rain water at physiological maturity. The test materials were grown in all environments in an augmented design, with one of the four controls appearing alternately after every nine rows. The experiments were planted in ridge and furrow systems, with row-to-row spacing of 60 and $10 \mathrm{~cm}$ between plants. The basal dose of inorganic fertilizers included $18 \mathrm{~kg} \mathrm{~N}$ and $46 \mathrm{~kg} \mathrm{P}_{2} \mathrm{O}_{5} \mathrm{ha}^{-1}$. The experiments were protected against pod borer (Helicoverpa armigera) damage. Two sprays of Acephate $75 \%$ SP. (Asataf) at $3 \mathrm{~g} \mathrm{~L}^{-1}$ water before flowering and three sprays of methomyl $40 \%$ SP. (Lannate) at $3 \mathrm{~g} \mathrm{~L}^{-1}$ of water from flowering to physiological maturity were given during the crop seasons. The plot size was 
Table 1. Weather data recorded during the period chickpea mini core trials were conducted, 2000 and 2001 to 2003 and 2004 crop seasons, Patancheru, India.

\begin{tabular}{|c|c|c|c|}
\hline $\begin{array}{c}\text { Weather } \\
\text { parameters }\end{array}$ & 2000-2001 & 2001-2002 & 2003-2004 \\
\hline Rainfall (mm) & 23.09 & 31.38 & 106.18 \\
\hline Evaporation (mm) & 686.61 & 648.23 & 559.87 \\
\hline Minimum temperature $\left({ }^{\circ} \mathrm{C}\right)$ & $13.57(9.11-17.51)$ & $13.68(9.24-17.35)$ & $14.48(10.37-19.72)$ \\
\hline Maximum temperature $\left({ }^{\circ} \mathrm{C}\right)$ & $30.4(26.45-34.54)$ & $29.62(26.17-33.44)$ & $28.72(26.19-33.17)$ \\
\hline Relative humidity at 07:17 (\%) & 85.89 (68.28-92.71) & 87.2 (69.71-93.71) & 90.66 (78.71-95.57) \\
\hline Relative humidity at $14: 17$ (\%) & $29.96(17.57-54.85)$ & $35.57(21.14-47.57)$ & $40.48(25.14-62.42)$ \\
\hline Wind velocity (Km h-1) & $4.8(2.75-7.52)$ & $5.33(2.47-12.01)$ & $5.17(3.24-7.75)$ \\
\hline Solar radiation (mJ m²) & $16.76(11.81-20.05)$ & $16.5(14.01-20.21)$ & $15.06(10.6-20.09)$ \\
\hline Bright sunshine (hr) & $9.26(6.31-10.51)$ & $9.11(7.62-10.29)$ & $8.64(5.54-10.58)$ \\
\hline
\end{tabular}

${ }^{\dagger}$ Cumulative rainfall and evaporation; mean and range (parentheses) represent temperature, relative humidity, wild velocity, solar radiation, and bright sunshine hours.

Table 2. Sources of resistance to abiotic and biotic stresses as reported by various workers after evaluating the chickpea mini core collection.

\begin{tabular}{|c|c|c|c|}
\hline \multirow[b]{2}{*}{ Stress } & \multicolumn{2}{|l|}{ Resistant genotype } & \multirow[b]{2}{*}{ Reference } \\
\hline & Desi & Kabuli & \\
\hline Drought & $\begin{array}{c}\text { ICC } 283,456,637,708,867,1205,1422,1431,1882 \\
\text { 2263, 2580, 3325, 4495, 4593, 5613, 5878, 6874, 7441, } \\
\text { 8950, 10399, 10945, 11121, 11944, 12155, 12947, 13124, } \\
\text { 14402, 14778, 14799, 14815, 15868, } 16524\end{array}$ & $\begin{array}{l}\text { ICC } 4872,5337 \\
7272,7323,8261 \\
16796\end{array}$ & $\begin{array}{l}\text { Kashiwagi et al., 2005, 2006b, 2008, 2010; } \\
\text { Parameshwarappa and Salimath, 2008; } \\
\text { Krishnamurthy et al., 2010; Mulwa et al., } \\
\text { 2010; Zaman-Allah et al., 2011a, 2011b }\end{array}$ \\
\hline Salinity & $\begin{array}{c}\text { ICC } 283,456,708,867,1431,2263,2580,3325,4495 \\
\begin{array}{c}4593,5613,5878,6279,6874,7441,9942,10399 \\
10945,11121,11944,12155,13124,14402,14778 \\
14799,15868,16524\end{array}\end{array}$ & $\begin{array}{l}\text { ICC 4872, } 7272 \\
8261,16796\end{array}$ & $\begin{array}{l}\text { Serraj et al., 2004; Vadez et al., 2007; } \\
\text { Krishnamurthy et al., 2011b }\end{array}$ \\
\hline Heat & $\begin{array}{c}\text { ICC } 283,456,637,708,1205,1882,2263,4495,5613 \\
5878,6874,7441,10945,11121,11944,12155,13124 \\
14402,14778,14799,14815,15868\end{array}$ & - & $\begin{array}{l}\text { Krishnamurthy et al., 2011a; Upadhyaya } \\
\text { et al., } 2011\end{array}$ \\
\hline Fusarium wilt & $\begin{array}{c}\text { ICC } 1710,1915,2242,2990,3325,4533,5135,6279 \\
6874,7184,7554,7819,12028,12155,13219,13599 \\
14402,14831,15606,15610\end{array}$ & $\begin{array}{c}\text { ICC } 2277,9848 \\
12037,13441,13816 \\
14199\end{array}$ & Pande et al., 2006 \\
\hline Dry root rot & ICC 1710, 2242 & $\begin{array}{c}\text { ICC } 2277,11764 \\
12328,13441\end{array}$ & Pande et al., 2006 \\
\hline Ascochyta blight & ICC 1915, 7184, 11284 & - & Pande et al., 2006 \\
\hline Botrytis gray mold & $\begin{array}{c}\text { ICC 2990, 4533, 6279, 7554, 7819, 11284, } 12028 \\
12155,13219,13599,15606,15610\end{array}$ & $\begin{array}{l}\text { ICC } 9848,11764 \\
12037,12328,13816 \\
14199,15406\end{array}$ & Pande et al., 2006 \\
\hline Legume pod borer & ICC 3325, 5135, 6874, 14402, 14831, 15606 & ICC 15406 & ICRISAT 2009; Mulwa et al., 2010 \\
\hline Herbicide & ICC 2242, 2580, 3325 & - & Taran et al., 2010 \\
\hline
\end{tabular}

$4.8 \mathrm{~m}^{2}$ under irrigated and $2.4 \mathrm{~m}^{2}$ under non-irrigated conditions. Data on days to $50 \%$ flower, flowering duration (days), 100-grain weight $(\mathrm{g})$, and grain yield $\left(\mathrm{kg} \mathrm{ha}^{-1}\right)$ were recorded on a plot basis. The environment-wise genetic variance and pooled genetic and genotype $\times$ environment interaction variances were estimated using Residual Maximum Likelihood (REML), on GENSTAT software (VSN International, 2013).

The published information on the performance of chickpea mini core accessions reporting resistance to abiotic and/or biotic stress (Table 2) was superimposed on agronomic data (days to $50 \%$ flower, flowering duration, 100-grain weight, and grain yield) to divide accessions into three distinct groups: abiotic stress resistant group (40 accessions), biotic stress resistant group (31 accessions), and a susceptible but agronomically desirable group (yielding at par or superior to controls; 24 accessions). Six of these accessions (ICC 2580, 3325, 6279, 6874, 12155, and
14402) were common as these were reported to be resistant to both abiotic and biotic stresses. The mini core accessions were part of a composite collection (3000 accessions) of chickpea (Upadhyaya et al., 2006), which was genotyped with 48 SSRs (Upadhyaya et al., 2008). A simple matching allele frequencybased distance matrix in DARwin 5.0 (Perrier et al., 2003), based on 48 SSR loci data on 93 accessions, was used to construct a neighbor-joining tree diagram and identify genetically diverse accessions with agronomically beneficial traits.

\section{RESULTS}

The REML analysis of individual environments (data not provided) and pooled analysis across seven environments detected highly significant genotypic variance $\left(\sigma^{2} g\right)$ for days to 50\% flower, flowering duration, 100-grain weight, 
Table 3. Variance components due to genotype $\left(\sigma^{2} g\right)$ and genotype $\times$ environment $\left(\sigma^{2} g\right.$ e) interaction in chickpea mini core collection accessions evaluated for four agronomic traits during the 2000 and 2001 to 2003 and 2004 post-rainy seasons at Patancheru, India.

\begin{tabular}{|c|c|c|c|c|c|c|}
\hline \multirow[t]{2}{*}{ Traits } & \multicolumn{2}{|c|}{$\begin{array}{l}\text { Pooled } 7 \\
\text { seasons }\end{array}$} & \multicolumn{2}{|c|}{$\begin{array}{l}\text { Pooled } 4 \\
\text { irrigated } \\
\text { seasons }\end{array}$} & \multicolumn{2}{|c|}{$\begin{array}{c}\text { Pooled } 3 \\
\text { non-irrigated } \\
\text { seasons }\end{array}$} \\
\hline & $\sigma^{2} g$ & $\sigma^{2} g e$ & $\sigma^{2} g$ & $\sigma^{2} \mathrm{ge}$ & $\sigma^{2} g$ & $\sigma^{2} \mathrm{ge}$ \\
\hline $\begin{array}{l}\text { Days to } 50 \% \\
\text { flowering }\end{array}$ & $84.01^{\star *}$ & $10.48^{\text {** }}$ & $81.10^{* *}$ & $12.57^{\star \star}$ & $86.55^{\star \star}$ & 4.16 \\
\hline $\begin{array}{c}\text { Flowering } \\
\text { duration }\end{array}$ & $10.66^{\star *}$ & $7.139^{\star \star}$ & $15.79^{\star \star}$ & $7.68^{\star \star}$ & $5.63^{\text {** }}$ & 0.93 \\
\hline $\begin{array}{l}\text { 100-grain } \\
\text { weight (g) }\end{array}$ & $45.24^{\star \star}$ & $1.38^{* *}$ & $45.66^{\star \star}$ & $1.76^{\star \star}$ & $46.20^{\star \star}$ & $0.45^{\star}$ \\
\hline $\begin{array}{c}\text { Grain yield } \\
\left(\mathrm{kg} \mathrm{ha}^{-1}\right)\end{array}$ & $36304^{\star *}$ & $14430^{\star *}$ & $35220^{\star *}$ & 3656 & $40391^{\star \star}$ & $13893^{*}$ \\
\hline
\end{tabular}

* Significant at the 0.05 probability level.

** Significant at the 0.01 probability level.

and grain yield, which indicate that sufficient genetic variations exist for these traits in chickpea mini core accessions (Table 3). Significant genotype $\times$ environment $\left(\sigma^{2}\right.$ ge) interaction across seven environments as well across four irrigated and three non-irrigated environments highlights the specific adaptation and sensitivity of traits to the environmental variations. Genotypic variance $\left(\sigma^{2} \mathrm{~g}\right)$ was also found highly significant for all of the four traits in irrigated and non-irrigated environments, while genotype $\times$ environment $\left(\sigma^{2}\right.$ ge) was significant for days to $50 \%$ flowering, flowering duration, and 100-grain weight in irrigated environments but only for 100-grain weight and grain yield in non-irrigated environments (Table 3).

The mean grain yield of 40 abiotic stress resistant accessions across seven environments ranged between 701 and $1656 \mathrm{~kg} \mathrm{ha}{ }^{-1}$, with ICC 15868 and ICC 14402 recording 12 to $18 \%$ higher grain yield over the best control, Annigeri (1407 kg ha-1; Table 4). ICC 637, 7441, and 15868, among the desi types under irrigated environments, produced 11 to $15 \%$ greater grain yield than Annigeri (average grain yield $1504 \mathrm{~kg} \mathrm{ha}^{-1}$ ). ICC 14402, across nonirrigated environments, recorded $27 \%$ greater grain yield than Annigeri (1303 $\left.\mathrm{kg} \mathrm{ha}^{-1}\right)$. ICC 7441, 14402, and 15868 combine resistance to terminal drought, salinity, and heat stress, while ICC 637 had resistance to terminal drought and heat stress (Kashiwagi et al., 2005, 2010; Vadez et al., 2007; Parameshwarappa and Salimath, 2008; Mulwa et al., 2010; Krishnamurthy et al., 2010, 2011a, 2011b; Upadhyaya

Table 4. Abiotic stress tolerant subset of the chickpea mini core accessions for variation in days to $50 \%$ flowering, flowering duration, 100-grain weight, and grain yield, evaluated during the 2000 and 2001 to 2003 and 2004 post-rainy seasons at Patancheru, India.

\begin{tabular}{|c|c|c|c|c|c|c|c|}
\hline \multirow[b]{2}{*}{$\begin{array}{l}\text { Germplasm } \\
\text { identity }\end{array}$} & \multirow[b]{2}{*}{$\begin{array}{l}\text { Country } \\
\text { of origin }\end{array}$} & \multirow[b]{2}{*}{$\begin{array}{l}\text { Days to } 50 \% \\
\text { flowering }\end{array}$} & \multirow[b]{2}{*}{$\begin{array}{l}\text { Flowering } \\
\text { duration }\end{array}$} & \multirow[b]{2}{*}{$\begin{array}{l}\text { 100-grain } \\
\text { weight }\end{array}$} & \multicolumn{3}{|c|}{ Grain yield } \\
\hline & & & & & $\begin{array}{l}\text { Irrigated } \\
\text { (4 seasons) }\end{array}$ & $\begin{array}{l}\text { Non-irrigated } \\
\text { (3 seasons) }\end{array}$ & $\begin{array}{c}\text { Pooled } \\
\text { (7 seasons) }\end{array}$ \\
\hline & & & $\begin{array}{c}d \\
\text { Desi chickpea }\end{array}$ & g & 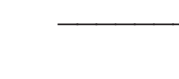 & $\mathrm{kg} \mathrm{ha}^{-1}$ & - \\
\hline ICC 283 & India & 57 & 33 & 14.8 & 1509 & 1140 & 1363 \\
\hline ICC 456 & India & 59 & 34 & 11.5 & 1418 & 1193 & 1327 \\
\hline ICC 637 & India & 54 & 35 & 15.0 & 1685 & 1008 & 1438 \\
\hline ICC 708 & India & 61 & 33 & 13.5 & 1328 & 1129 & 1232 \\
\hline ICC 867 & India & 53 & 34 & 16.0 & 1315 & 1163 & 1237 \\
\hline ICC 1205 & India & 58 & 34 & 15.6 & 1578 & 853 & 1285 \\
\hline ICC 1422 & India & 47 & 35 & 18.5 & 1327 & 1134 & 1240 \\
\hline ICC 1431 & India & 61 & 31 & 17.3 & 1451 & 1009 & 1265 \\
\hline ICC 1882 & India & 50 & 35 & 15.8 & 1297 & 1281 & 1306 \\
\hline ICC 2263 & Iran & 60 & 31 & 14.7 & 1371 & 1104 & 1274 \\
\hline ICC 2580 & Iran & 50 & 37 & 19.0 & 1549 & 1107 & 1406 \\
\hline ICC 3325 & Cyprus & 56 & 34 & 15.0 & 1583 & 1334 & 1535 \\
\hline ICC 4495 & Turkey & 58 & 33 & 13.2 & 1431 & 1118 & 1315 \\
\hline ICC 4593 & India & 59 & 33 & 15.3 & 1365 & 1216 & 1309 \\
\hline ICC 5613 & India & 49 & 35 & 16.2 & 1366 & 919 & 1175 \\
\hline ICC 5878 & India & 51 & 35 & 11.2 & 1547 & 941 & 1316 \\
\hline ICC 6279 & India & 46 & 37 & 15.0 & 1477 & 1138 & 1351 \\
\hline ICC 6874 & Iran & 59 & 34 & 12.9 & 1492 & 1115 & 1358 \\
\hline ICC 7441 & India & 50 & 35 & 14.1 & 1664 & 1177 & 1507 \\
\hline ICC 8950 & India & 57 & 34 & 13.0 & 1451 & 1287 & 1405 \\
\hline ICC 9942 & India & 57 & 34 & 11.6 & 1240 & 1049 & 1135 \\
\hline ICC 10399 & India & 49 & 37 & 14.9 & 1618 & 1150 & 1454 \\
\hline ICC 10945 & India & 52 & 34 & 13.9 & 1519 & 1187 & 1402 \\
\hline ICC 11121 & India & 57 & 33 & 12.1 & 1472 & 1113 & 1338 \\
\hline
\end{tabular}




\begin{tabular}{|c|c|c|c|c|c|c|c|}
\hline \multirow[b]{2}{*}{$\begin{array}{l}\text { Germplasm } \\
\text { identity }\end{array}$} & \multirow[b]{2}{*}{$\begin{array}{l}\text { Country } \\
\text { of origin }\end{array}$} & \multirow[b]{2}{*}{$\begin{array}{c}\text { Days to } 50 \% \\
\text { flowering }\end{array}$} & \multirow[b]{2}{*}{$\begin{array}{c}\text { Flowering } \\
\text { duration }\end{array}$} & \multirow[b]{2}{*}{$\begin{array}{c}\text { 100-grain } \\
\text { weight }\end{array}$} & \multicolumn{3}{|c|}{ Grain yield } \\
\hline & & & & & $\begin{array}{c}\text { Irrigated } \\
\text { (4 seasons) }\end{array}$ & $\begin{array}{c}\text { Non-irrigated } \\
\text { (3 seasons) }\end{array}$ & $\begin{array}{c}\text { Pooled } \\
\text { (7 seasons) }\end{array}$ \\
\hline ICC 11944 & Nepal & 63 & 31 & 12.4 & 1442 & 1154 & 1332 \\
\hline ICC 12155 & Bangladesh & 56 & 34 & 13.4 & 1412 & 1194 & 1331 \\
\hline ICC 12947 & India & 65 & 31 & 16.5 & 1363 & 1121 & 1264 \\
\hline ICC 13124 & India & 43 & 35 & 31.5 & 1427 & 1167 & 1329 \\
\hline ICC 14402 & India & 46 & 35 & 15.9 & 1569 & 1652 & 1656 \\
\hline ICC 14778 & India & 58 & 33 & 12.4 & 1546 & 1059 & 1361 \\
\hline ICC 14799 & India & 61 & 30 & 13.9 & 1505 & 1034 & 1322 \\
\hline ICC 14815 & India & 58 & 35 & 14.6 & 1535 & 1288 & 1459 \\
\hline ICC 15868 & India & 55 & 33 & 12.4 & 1729 & 1224 & 1572 \\
\hline ICC 16524 & Pakistan & 56 & 34 & 14.4 & 1430 & 1283 & 1390 \\
\hline Entry mean (desi) & & 55 & 34 & 15.0 & 1471 & 1148 & 1353 \\
\hline \multicolumn{8}{|l|}{ Trial control (desi) } \\
\hline Annigeri & India & 46 & 37 & 21.5 & 1504 & 1303 & 1407 \\
\hline G 130 & India & 63 & 33 & 13.2 & 1525 & 1172 & 1393 \\
\hline Trial control mean (de & & 55 & 35 & 17.0 & 1515 & 1237 & 1400 \\
\hline \multicolumn{8}{|c|}{ Kabuli chickpea } \\
\hline ICC 5337 & India & 75 & 27 & 23.8 & 1137 & 641 & 887 \\
\hline ICC 7272 & Algeria & 59 & 37 & 29.9 & 1276 & 896 & 1104 \\
\hline ICC 8261 & Turkey & 64 & 30 & 32.8 & 1138 & 936 & 1026 \\
\hline ICC 16796 & Portugal & 74 & 28 & 37.9 & 898 & 613 & 701 \\
\hline ICC 4872 & India & 46 & 36 & 19.8 & 1373 & 1112 & 1267 \\
\hline ICC 7323 & Russian Federation & 65 & 34 & 20.3 & 961 & 619 & 751 \\
\hline Entry mean (kabuli) & & 64 & 32 & 27.0 & 1131 & 803 & 956 \\
\hline \multicolumn{8}{|l|}{ Trial control (kabuli) } \\
\hline L 550 & India & 64 & 33 & 20.0 & 1407 & 1042 & 1262 \\
\hline ICCV 2 & India & 45 & 27 & 20.2 & 1240 & 1019 & 1111 \\
\hline Trial control mean (ka & & 54 & 30 & 20.0 & 1324 & 1030 & 1187 \\
\hline 40 entry mean (desi & kabuli types) & 56 & 34 & 16.8 & 1420 & 1096 & 1293 \\
\hline Trial mean ${ }^{\dagger}$ & & 60.49 & 31.34 & 17.3 & 1239 & 860.7 & 1082 \\
\hline Trial range $^{\dagger}$ & & $41-83$ & $24-46$ & $10-43$ & $858-1731$ & $575-1652$ & $701-1656$ \\
\hline $\mathrm{SE}^{\ddagger} \pm$ & & 2.719 & 1.956 & 1.051 & 151.8 & 163.7 & 134.1 \\
\hline LSD (5\%) & & 7.541 & 5.425 & 2.915 & 421.1 & 454.3 & 371.9 \\
\hline $\mathrm{CV}^{\ddagger}(\%)$ & & 7.146 & 13.01 & 8.854 & 29.72 & 32.79 & 30.76 \\
\hline
\end{tabular}

$\dagger$ Indicate mean and range for the entire trial.

‡SE, standard error; LSD least significant difference; CV, coefficient of variation.

et al., 2011). ICC 14402 flowered at the same time as Annigeri (46 d), while ICC 15868 flowered 9 d later than Annigeri. Another drought, salinity, and heat stress resistant line, ICC 13124 (Kashiwagi et al., 2010; Vadez et al., 2007; Krishnamurthy et al., 2010, 2011a, 2011b; Upadhyaya et al., 2011), flowered $3 \mathrm{~d}$ earlier than Annigeri (46 d). It had larger seed size (32 g compared to $22 \mathrm{~g}$ of Annigeri); however, it yielded similarly (mean grain yield 1167 to $1427 \mathrm{~kg}$ $\mathrm{ha}^{-1}$ ) to Annigeri (mean grain yield $1303-1504 \mathrm{~kg} \mathrm{ha}^{-1}$ ) under both irrigated and non-irrigated environments. Some of the other accessions, combining terminal drought, salinity, and heat stress resistances or possessing resistance only to terminal drought, yielded similarly to Annigeri (average yield across seven environments: 1402 to $1535 \mathrm{~kg}$ $\mathrm{ha}^{-1}$; Annigeri: $1407 \mathrm{~kg} \mathrm{ha}^{-1}$ ). None of the kabuli types, except ICC 4872 (7\% more in non-irrigated and on par in pooled across 7 environments), were found high yielding in either irrigated or non-irrigated environments or across seven environments in comparison to the best control, L550 (mean grain yield 1042-1407 $\mathrm{kg} \mathrm{ha}^{-1}$ ). ICC 4872 is reported resistant to drought and salinity (Kashiwagi et al., 2005, 2010; Krishnamurthy et al., 2011b). The drought and salinity resistant accessions, ICC 7272, 8261, and 16796 (Krishnamurthy et al., 2010, 2011b; Kashiwagi et al., 2005, 2010), had higher 100-grain weight (30-38 g) compared to L 550 (20 g). Likewise, ICC 5337 combines high $\Delta^{13} \mathrm{C}$, transpiration efficiency, and root length density, all conferring resistance to drought (Kashiwagi et al., 2005, 2006a, 2010; Zaman-Allah et al., 2011a, 2011b); however, it produced relatively low yield across environments (Table 4).

The mean grain yield of 31 biotic stress resistant accessions across seven environments ranged between 842 and 
Table 5. Biotic stress resistant subset of the chickpea mini core accessions for variation in days to $50 \%$ flowering, flowering duration, 100-grain weight, and grain yield, evaluated during the 2000 and 2001 to 2003 and 2004 post-rainy seasons at Patancheru, India.

\begin{tabular}{|c|c|c|c|c|c|c|c|}
\hline \multirow[b]{2}{*}{ Germplasm identity } & \multirow[b]{2}{*}{ Country of origin } & \multirow[b]{2}{*}{$\begin{array}{c}\text { Days to } 50 \% \\
\text { flowering }\end{array}$} & \multirow[b]{2}{*}{$\begin{array}{c}\text { Flowering } \\
\text { duration }\end{array}$} & \multirow[b]{2}{*}{$\begin{array}{l}\text { 100-grain } \\
\text { weight }\end{array}$} & \multicolumn{3}{|c|}{ Grain yield } \\
\hline & & & & & $\begin{array}{c}\text { Irrigated } \\
\text { (4 seasons) }\end{array}$ & $\begin{array}{c}\text { Non-irrigated } \\
\text { (3 seasons) }\end{array}$ & $\begin{array}{c}\text { Pooled } \\
\text { (7 seasons) }\end{array}$ \\
\hline & & & $\begin{array}{c}d \\
\text { Desi chickp }\end{array}$ & $g$ & 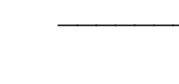 & $\mathrm{kg} \mathrm{ha}^{-1}$ & -2 \\
\hline ICC 1710 & India & 68 & 31 & 12.2 & 1449 & 1154 & 1357 \\
\hline ICC 1915 & India & 79 & 26 & 25.5 & 1143 & 575 & 869 \\
\hline ICC 2242 & India & 77 & 28 & 12.9 & 1463 & 673 & 1150 \\
\hline ICC 2580 & Iran & 50 & 37 & 19 & 1549 & 1107 & 1406 \\
\hline ICC 2990 & Iran & 64 & 33 & 19.1 & 1277 & 818 & 1080 \\
\hline ICC 3325 & Cyprus & 56 & 34 & 15 & 1583 & 1334 & 1535 \\
\hline ICC 4533 & India & 43 & 35 & 17.9 & 1289 & 1074 & 1193 \\
\hline ICC 5135 & India & 67 & 30 & 12.2 & 1610 & 864 & 1331 \\
\hline ICC 6279 & India & 46 & 37 & 15 & 1477 & 1138 & 1351 \\
\hline ICC 6874 & Iran & 59 & 34 & 12.9 & 1492 & 1115 & 1358 \\
\hline ICC 7184 & Turkey & 63 & 34 & 9.7 & 1247 & 1009 & 1138 \\
\hline ICC 7554 & Iran & 63 & 34 & 24.2 & 1319 & 957 & 1167 \\
\hline ICC 7819 & Iran & 66 & 33 & 21.2 & 1176 & 852 & 1005 \\
\hline ICC 11284 & Russian Federation & 70 & 31 & 15.4 & 1039 & 720 & 844 \\
\hline ICC 12028 & Mexico & 63 & 33 & 22 & 1289 & 874 & 1094 \\
\hline ICC 12155 & Bangladesh & 56 & 34 & 13.4 & 1412 & 1194 & 1331 \\
\hline ICC 13219 & Iran & 52 & 32 & 14 & 1466 & 1202 & 1378 \\
\hline ICC 13599 & Iran & 67 & 33 & 22.3 & 1339 & 797 & 1101 \\
\hline ICC 14402 & India & 46 & 35 & 15.9 & 1569 & 1652 & 1656 \\
\hline ICC 14831 & India & 59 & 34 & 13.6 & 1472 & 1171 & 1369 \\
\hline ICC 15606 & India & 53 & 34 & 15.1 & 1522 & 1086 & 1366 \\
\hline ICC 15610 & India & 72 & 29 & 19.7 & 1520 & 914 & 1284 \\
\hline Entry mean (desi) & & 61 & 33 & 17 & 1396 & 1013 & 1244 \\
\hline \multicolumn{8}{|l|}{ Trial Control (desi) } \\
\hline Annigeri & India & 46 & 37 & 21.5 & 1504 & 1303 & 1407 \\
\hline G 130 & India & 63 & 33 & 13.2 & 1525 & 1172 & 1393 \\
\hline Trial control mean (desi) & & 55 & 35 & 17 & 1515 & 1237 & 1400 \\
\hline \multicolumn{8}{|c|}{ Kabuli chickpea } \\
\hline ICC 2277 & Iran & 73 & 29 & 23.2 & 1163 & 816 & 1000 \\
\hline ICC 9848 & Afghanistan & 59 & 34 & 16.5 & 1387 & 863 & 1165 \\
\hline ICC 11764 & Chile & 73 & 28 & 29.4 & 1325 & 748 & 1067 \\
\hline ICC 12037 & Mexico & 68 & 31 & 18.2 & 1191 & 831 & 1010 \\
\hline ICC 12328 & Cyprus & 71 & 29 & 34.8 & 1243 & 750 & 1006 \\
\hline ICC 13441 & Iran & 78 & 26 & 17.1 & 1298 & 820 & 1082 \\
\hline ICC 13816 & Russian Federation & 73 & 28 & 24.9 & 1374 & 770 & 1111 \\
\hline ICC 14199 & Mexico & 60 & 33 & 43.2 & 1076 & 650 & 842 \\
\hline ICC 15406 & Morocco & 70 & 29 & 36.1 & 1146 & 862 & 990 \\
\hline Entry mean (kabuli) & & 70 & 30 & 27 & 1245 & 790 & 1030 \\
\hline \multicolumn{8}{|l|}{ Trial control (kabuli) } \\
\hline L 550 & India & 64 & 33 & 20 & 1407 & 1042 & 1262 \\
\hline ICCV 2 & India & 45 & 27 & 20.2 & 1240 & 1019 & 1111 \\
\hline \multicolumn{2}{|l|}{ Trial control mean (kabuli) } & 54 & 30 & 20 & 1324 & 1030 & 1187 \\
\hline \multicolumn{2}{|c|}{31 entry mean (desi andkabuli types) } & 63 & 32 & 19.7 & 1352 & 948 & 1182 \\
\hline \multicolumn{2}{|l|}{ Trial mean ${ }^{\dagger}$} & 60.49 & 31.34 & 17.3 & 1239 & 860.7 & 1082 \\
\hline \multicolumn{2}{|l|}{ Trial range $^{\dagger}$} & $41-83$ & $24-46$ & $10-43$ & $858-1731$ & $575-1652$ & $701-1656$ \\
\hline \multicolumn{2}{|l|}{$\mathrm{SE}^{\ddagger} \pm$} & 2.719 & 1.956 & 1.051 & 151.8 & 163.7 & 134.1 \\
\hline \multicolumn{2}{|l|}{ LSD (5\%) $^{2}$} & 7.541 & 5.425 & 2.915 & 421.1 & 454.3 & 371.9 \\
\hline \multicolumn{2}{|l|}{ CV₹ (\%) } & 7.146 & 13.01 & 8.854 & 29.72 & 32.79 & 30.76 \\
\hline
\end{tabular}

$\dagger$ Indicate mean and range for the entire trial.

‡SE, standard error; LSD least significant difference; CV, coefficient of variation. 
$1656 \mathrm{~kg} \mathrm{ha}^{-1}$ (Table 5). ICC 5135 recorded 7\% greater grain yield than Annigeri under irrigated environments, while ICC 3325 and ICC 14402, across seven environments, produced 9 to $18 \%$ higher grain yield compared to Annigeri (mean grain yield $1504 \mathrm{~kg} \mathrm{ha}^{-1}$ under irrigated and $1407 \mathrm{~kg} \mathrm{ha}^{-1}$ across seven environments). All three accessions were reported resistant to fusarium wilt (FW; Pande et al., 2006), ICC 3325 and ICC 5135 to legume pod borer [LPB]) (ICRISAT, 2009; Mulwa et al., 2010), while ICC 3325 to Odyssey (BASF, Canada) herbicide (containing imazethapyr and imazamox herbicides, 35\% each at $30 \mathrm{~g}$ a.i. $\mathrm{ha}^{-1}$; Taran et al., 2010). A number of desi type accessions recorded greater grain yield over the entry mean of their group. For example, ICC 2580, 3325, $5135,14402,15606$, and 15610 showed a 9 to $15 \%$ yield advantage under irrigated environments and ICC 3325, 12155,14402 , and 13219 showed a 18 to $63 \%$ yield advantage under non-irrigated environments. ICC 3325 and ICC 14402 produced 12 to $13 \%, 32$ to $63 \%$, and 23 to $33 \%$ greater grain yield against entry means $\left(1396 \mathrm{~kg} \mathrm{ha}^{-1}\right.$ under irrigated environments, $1013 \mathrm{~kg} \mathrm{ha}^{-1}$ under nonirrigated environments, and $1244 \mathrm{~kg} \mathrm{ha}^{-1}$ across seven environments) across irrigated, non-irrigated, or across both environments. ICC 12155, 13219, 15606, and 15610 were reported resistant to FW and botrytis gray mold (BGM; Pande et al., 2006), ICC 15606 to LPB (ICRISAT, 2009; Mulwa et al., 2010), and ICC 2580 and ICC 3325 to herbicide (Taran et al., 2010). None of the kabuli accessions in this group yielded greater than the best control, L550 (average grain yield across seven environments, 1262 $\left.\mathrm{kg} \mathrm{ha}^{-1}\right)$. However, ICC 11764, 12328, 14199, and 15406 showed greater 100-grain weight (29-43 g) compared to L 550 (20 g 100 ${ }^{-1}$ grains). ICC 11764 and ICC 12328 were reported resistant to BGM and dry root rot (DRR); ICC 14199 to FW and BGM; and ICC 15406 to BGM and LPB (Pande et al., 2006; ICRISAT, 2009; Mulwa et al., 2010).

The mean grain yield of 24 accessions agronomically desirable but susceptible to abiotic and biotic stress, across seven environments ranged between 1006 and $1506 \mathrm{~kg}$ $\mathrm{ha}^{-1}$ (Table 6). None of these accessions, either desi or kabuli types, produced significantly greater yield than the best controls. However, ICC 1098, 9586, and 12307 among desi types produced on average 11 to $15 \%$ greater grain yield than Annigeri (mean grain yield $1504 \mathrm{~kg} \mathrm{ha}^{-1}$ ) in irrigated environments. Kabuli chickpea accession, ICC 7668, produced average grain yield of $1253 \mathrm{~kg} \mathrm{ha}^{-1}$, comparable to the best control, L $550\left(1262 \mathrm{~kg} \mathrm{ha}^{-1}\right)$. Averaged across seven environments, most of the desi types yielded approximately $1400 \mathrm{~kg} \mathrm{ha}^{-1}$, while ICC 7255, 7668, 15333, and 15697, the kabuli types produced approximately $1200 \mathrm{~kg} \mathrm{ha}^{-1}$ and 100 -grain weight ranged from 26 to $38 \mathrm{~g}$, which is 30 to $90 \%$ greater than the best control, L 550 (20 g).

Germplasm with multiple resistant traits, both abiotic and biotic stress, offer breeders opportunities to develop breeding and genetic mapping populations combining multiple resistances into an agronomically improved genetic background. A number of chickpea mini core accessions were reported resistant to both abiotic and biotic stress. For example, a drought and salinity resistant accession, ICC 3325, possesses resistance to FW, LPB, and herbicide (Odyssey), while ICC 6874, 12155, and 14402, in addition to possessing resistance to drought, heat, and salinity, also combine resistance to FW, LPB, or BGM (Pande et al., 2006; Mulwa et al., 2010; ICRISAT 2009; Taran et al., 2010). Likewise, ICC 6279 is resistant to salinity, FW, and BGM, while ICC 2580 is resistant to drought, salinity, and herbicide. Of course, for complex constraints such as drought, we also have to consider the high likelihood of genotype $\times$ environment interactions, whereby the drought adapted genotypes selected here might not be suitable in all drought environments.

A neighbor-joining tree broadly separated the 93 accessions into four clusters (Fig. 1). All the accessions possessing no resistance to either abiotic or biotic stress grouped in cluster I (mostly kabuli types) and II (all desi types). The stress resistant accessions grouped in clusters III and IV. Cluster III had more representation of abiotic stress resistant accessions while cluster IV populated with biotic stress resistant accessions. The abiotic stress resistant accessions were genetically more diverse as evidenced by a higher range of genetic distance between accessions (range 0.717 amongst 780 pairs) compared to those in the biotic stress resistant group (range 0.585 amongst 465 pairs) (Table 7). A number of genetically diverse pairs with resistance to stress and agronomic performance similar to the controls have been identified for enhancing the trait values. For example, ICC 12155 (drought, heat, salinity, FW, BGM) and ICC 5337 (root length density [RLD], TE, and $\delta^{13}$ ); ICC 14402 (drought, heat, salinity, FW, LPB) with ICC 8261 (drought avoidance root traits, salinity, BGM), ICC 9848 (salinity, FW, BGM), and ICC 1915 (drought, FW, AB); ICC 3325 (drought, heat, salinity, herbicide, FW, LPB) with ICC 13816 (drought, salinity, FW, BGM), ICC 7554 (salinity, FW, BGM), ICC 9848 (drought, salinity, FW, BGM); and ICC 13599 (heat, salinity, FW, BGM, LPB).

\section{DISCUSSION}

Chickpea, like many other legumes, has a narrow genetic base due to bottlenecks associated with its domestication (Abbo et al., 2003). In addition, the crop suffers from many abiotic and biotic stresses causing substantial yield losses to production (Dwivedi et al., 2005). The crop is largely grown on marginal soils and poor crop management conditions. Chickpea does not respond to intensive crop management practices (high dose of fertilizers and more than two irrigations) to raise productivity as the crop has been developed under low input conditions (Smithson et al., 1985; Gaur et al., 2010). More importantly, since 
Table 6. Susceptible (abiotic and biotic stress) subset of the chickpea mini core accessions for variation in days to $50 \%$ flowering, flowering duration, 100-grain weight, and grain yield, evaluated during the 2000 and 2001 to 2003 and 2004 postrainy seasons at Patancheru, India.

\begin{tabular}{|c|c|c|c|c|c|c|c|}
\hline \multirow[b]{2}{*}{$\begin{array}{l}\text { Germplasm } \\
\text { identity }\end{array}$} & \multirow[b]{2}{*}{$\begin{array}{l}\text { Country } \\
\text { of origin }\end{array}$} & \multirow[b]{2}{*}{$\begin{array}{l}\text { Days to } 50 \% \\
\text { flowering }\end{array}$} & \multirow[b]{2}{*}{$\begin{array}{c}\text { Flowering } \\
\text { duration }\end{array}$} & \multirow[b]{2}{*}{$\begin{array}{c}\text { 100-grain } \\
\text { weight }\end{array}$} & \multicolumn{3}{|c|}{ Grain yield } \\
\hline & & & & & $\begin{array}{c}\text { Irrigated } \\
\text { (4 seasons) }\end{array}$ & $\begin{array}{c}\text { Non-irrigated } \\
\text { (3 seasons) }\end{array}$ & $\begin{array}{c}\text { Pooled } \\
\text { (7 seasons) }\end{array}$ \\
\hline & & & $\begin{array}{c}d \\
\text { Desi chickp }\end{array}$ & $g$ & 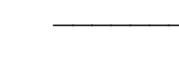 & $\mathrm{kg} \mathrm{ha}^{-1}$ & - \\
\hline ICC 440 & India & 63 & 31 & 11.8 & 1573 & 1277 & 1474 \\
\hline ICC 1098 & Iran & 58 & 31 & 13.1 & 1731 & 1074 & 1493 \\
\hline ICC 1180 & India & 66 & 30 & 15 & 1616 & 1167 & 1450 \\
\hline ICC 1230 & India & 51 & 34 & 20.7 & 1548 & 1141 & 1407 \\
\hline ICC 3362 & Iran & 56 & 34 & 11.5 & 1660 & 1100 & 1476 \\
\hline ICC 4567 & India & 56 & 35 & 20.6 & 1607 & 1123 & 1431 \\
\hline ICC 8384 & India & 51 & 35 & 14.5 & 1605 & 1204 & 1464 \\
\hline ICC 8621 & Ethiopia & 49 & 34 & 15.8 & 1589 & 1254 & 1484 \\
\hline ICC 9586 & India & 68 & 35 & 14.9 & 1687 & 947 & 1420 \\
\hline ICC 12307 & Myanmar & 56 & 34 & 12.1 & 1677 & 1160 & 1506 \\
\hline ICC 12866 & Ethiopia & 54 & 34 & 12.4 & 1522 & 1230 & 1418 \\
\hline ICC 14595 & India & 44 & 37 & 21.3 & 1161 & 1304 & 1195 \\
\hline ICC 15618 & India & 48 & 36 & 13.4 & 1523 & 1314 & 1467 \\
\hline ICC 16207 & Myanmar & 65 & 32 & 13.6 & 1548 & 1233 & 1453 \\
\hline ICC 16374 & Malawi & 41 & 46 & 20.4 & 1275 & 704 & 1006 \\
\hline Entry mean (desi) & & 55 & 34 & 15 & 1555 & 1149 & 1410 \\
\hline \multicolumn{8}{|l|}{ Trial control (desi) } \\
\hline Annigeri & India & 46 & 37 & 21.5 & 1504 & 1303 & 1407 \\
\hline G 130 & India & 63 & 33 & 13.2 & 1525 & 1172 & 1393 \\
\hline Trial control mean (d & & 55 & 35 & 17 & 1515 & 1237 & 1400 \\
\hline \multicolumn{8}{|c|}{ Kabuli chickpea } \\
\hline ICC 7255 & India & 59 & 33 & 30.4 & 1344 & 1078 & 1227 \\
\hline ICC 7315 & Iran & 61 & 33 & 30.9 & 1180 & 881 & 1022 \\
\hline ICC 7668 & Russian Federation & 58 & 34 & 25.9 & 1391 & 1038 & 1253 \\
\hline ICC 8151 & U.S.A. & 67 & 32 & 34.9 & 1298 & 987 & 1164 \\
\hline ICC 9137 & Iran & 68 & 32 & 35.5 & 1115 & 1024 & 1044 \\
\hline ICC 10755 & Turkey & 60 & 34 & 31.5 & 1198 & 839 & 1021 \\
\hline ICC 15333 & Iran & 63 & 35 & 32.3 & 1226 & 1259 & 1231 \\
\hline ICC 15518 & Morocco & 55 & 36 & 38.2 & 1198 & 909 & 1049 \\
\hline ICC 15697 & Syrian Arab Republic & 64 & 32 & 32.3 & 1321 & 1077 & 1210 \\
\hline Entry mean (kabuli) & & 62 & 33 & 32 & 1252 & 1010 & 1136 \\
\hline \multicolumn{8}{|l|}{ Trial control (kabuli) } \\
\hline L 550 & India & 64 & 33 & 20 & 1407 & 1042 & 1262 \\
\hline ICCV 2 & India & 45 & 27 & 20.2 & 1240 & 1019 & 1111 \\
\hline Trial control mean ( $\mathrm{k}$ & & 54 & 30 & 20 & 1324 & 1030 & 1187 \\
\hline 24 entry mean (desi & rabuli types) & 58 & 34 & 21.8 & 1441 & 1097 & 1307 \\
\hline Trial mean ${ }^{\dagger}$ & & 60.49 & 31.34 & 17.3 & 1239 & 860.7 & 1082 \\
\hline Trial range $^{\dagger}$ & & $41-83$ & $24-46$ & $10-43$ & $858-1731$ & $575-1652$ & $701-1656$ \\
\hline $\mathrm{SE}^{\ddagger} \pm$ & & 2.719 & 1.956 & 1.051 & 151.8 & 163.7 & 134.1 \\
\hline LSD (5\%) & & 7.54 & 5.43 & 2.92 & 421.1 & 454.3 & 371.9 \\
\hline $\mathrm{CV}^{\ddagger}(\%)$ & & 7.15 & 13.01 & 8.85 & 29.72 & 32.79 & 30.76 \\
\hline
\end{tabular}

† Indicate mean and range for the entire trial.

$\ddagger$ SE, standard error; LSD least significant difference; CV, coefficient of variation.

resource-poor chickpea farmers are not able to adopt intensive management practices, genetic enhancement including resistance to abiotic and biotic stresses is the way forward to increase and stabilize chickpea production, dominated by resource-poor farmers in Asia and Africa. Identification of genetically diverse germplasm with beneficial agronomic traits and their use in breeding will accelerate development of chickpea cultivars adapted to varied agro-ecological conditions.

Agronomic performance of stress resistant germplasm is a key factor in the choice of germplasm used in breeding programs. The chickpea mini core was evaluated for 


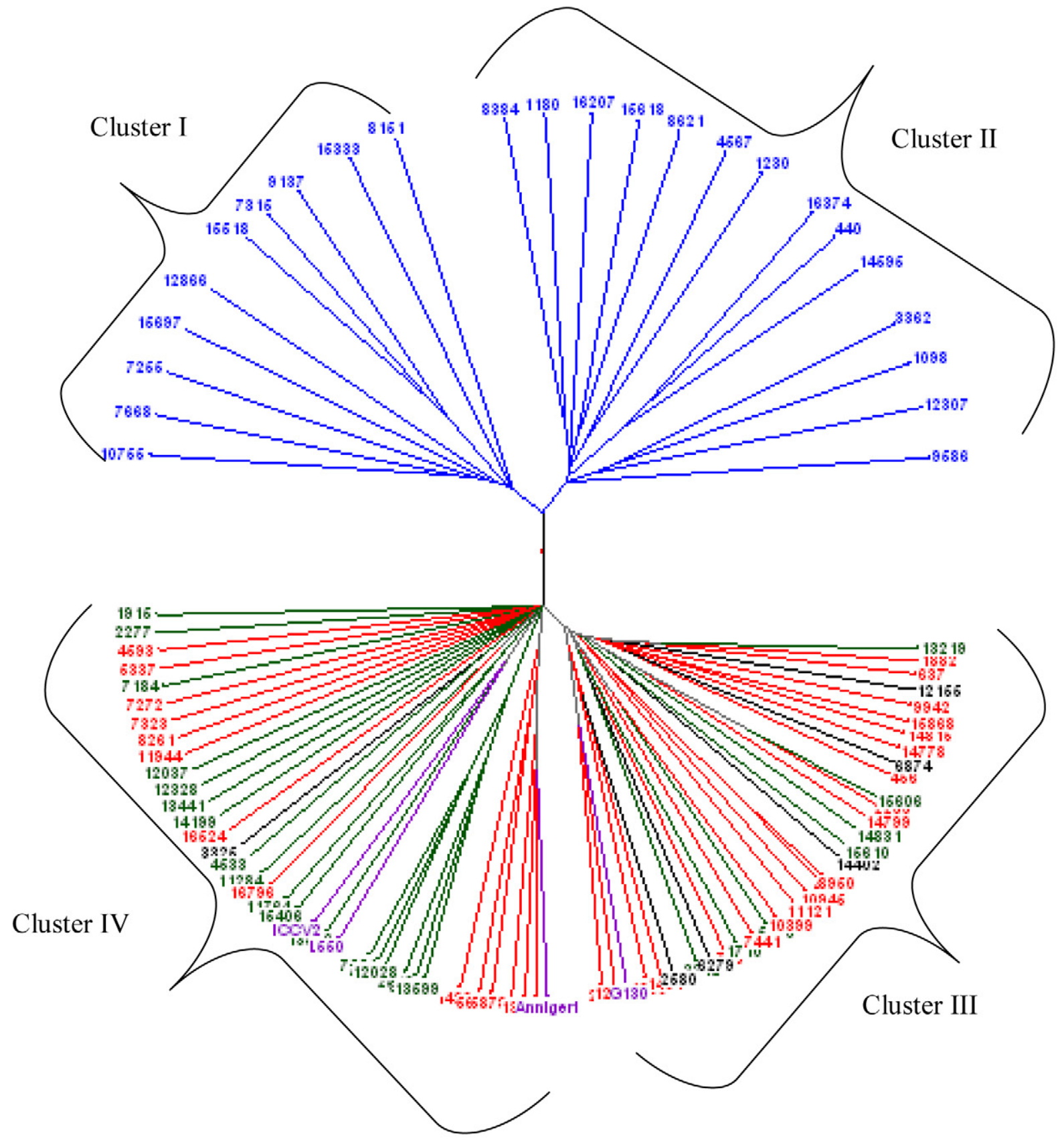

Red: abiotic resistance; Green: biotic resistance; Black: resistant to abiotic and biotic stress; Blue: susceptible to abiotic and biotic stress; Violet: controls

Figure 1. Clustering of 89 chickpea mini core accessions and four control cultivars into four groups: cluster I (susceptible kabuli types), cluster II (susceptible desi types), cluster III (mostly abiotic resistance), and cluster IV (mostly biotic stress resistance) based on simple matching distance matrix and unweighted pair group method with arithmetic mean (UPGMA) of 48 simple sequence repeat (SSR) marker data.

four key agronomic traits (days to $50 \%$ flowering, flowering duration, 100-grain weight, and grain yield) in four irrigated and three non-irrigated environments. In the desi group, the abiotic and/or biotic stress resistant accessions such as ICC 7441, 3325, 5135, 6874, 12155, 13219, 14402,15606 , and 15868 yielded similarly to the best control, Annigeri; however, their grain yield in many cases exceeded the mean grain yield of the desi types in the trial, with few accessions showing more specific adaptation to either irrigated or non-irrigated environments. None of the kabuli type accessions produced greater yield than the best control, L 550; however, many had 100-grain weight ranging between 30 and $43 \mathrm{~g}$, far greater than L 550 (20 g).

In the present study, the genotype-based distance matrix grouped the stress resistant germplasm separately, cluster III and IV, to those susceptible to stress, which grouped in cluster I and II (Fig. 1). Such a perfect grouping is seen only when there is a statistically significant 
Table 7. Twenty pairs of most genetically diverse (based on 48 SSR loci) chickpea mini core subset accessions selected amongst abiotic, biotic, and susceptible groups.

\begin{tabular}{|c|c|c|c|}
\hline Identity & $\begin{array}{l}\text { Genetic } \\
\text { distance }\end{array}$ & Identity & $\begin{array}{l}\text { Genetic } \\
\text { distance }\end{array}$ \\
\hline \multicolumn{4}{|c|}{ Abiotic stress tolerant group } \\
\hline ICC 16796 and ICC 283 & 1.000 & ICC 14402 and ICC 8261 & 0.969 \\
\hline ICC 9942 and ICC 7272 & 1.000 & ICC 5337 and ICC 1882 & 0.958 \\
\hline ICC 5337 and ICC 456 & 0.979 & ICC 8261 and ICC 2580 & 0.958 \\
\hline ICC 7272 and ICC 637 & 0.979 & ICC 5337 and ICC 4872 & 0.958 \\
\hline ICC 16796 and ICC 4495 & 0.979 & ICC 14778 and ICC 5337 & 0.958 \\
\hline ICC 12155 and ICC 5337 & 0.979 & ICC 16796 and ICC 1882 & 0.958 \\
\hline ICC 7272 and ICC 283 & 0.978 & ICC 7272 and ICC 708 & 0.957 \\
\hline ICC7323 and ICC 283 & 0.978 & ICC 7272 and ICC 4495 & 0.957 \\
\hline ICC 8261 and ICC 1205 & 0.978 & ICC 7272 and ICC 5337 & 0.957 \\
\hline ICC 7272 and ICC 1422 & 0.978 & ICC 15868 and ICC 5337 & 0.957 \\
\hline \multicolumn{4}{|c|}{$\begin{array}{l}\text { Minimum: } 0.283 \text { (ICC } 8950 \text { and ICC 4495); Maximum: } 1.000 \text { (ICC } 16796 \\
\text { and ICC 283); Range genetic distance: } 0.717 \text { amongst } 780 \text { pairs }\end{array}$} \\
\hline \multicolumn{4}{|c|}{ Biotic stress resistant group } \\
\hline ICC 13816 and ICC 3325 & 1.000 & ICC 7554 and ICC 3325 & 0.978 \\
\hline ICC 12328 and ICC 5135 & 1.000 & ICC 13816 and ICC 1710 & 0.977 \\
\hline ICC 15606 and ICC 9848 & 1.000 & ICC 2242 and ICC 1915 & 0.958 \\
\hline ICC 14402 and ICC 9848 & 0.979 & ICC 14402 and ICC 1915 & 0.958 \\
\hline ICC 15406 and ICC 14831 & 0.979 & ICC 12328 and ICC 2242 & 0.958 \\
\hline ICC 12155 and ICC 1915 & 0.979 & ICC 15406 and ICC 2242 & 0.958 \\
\hline ICC 9848 and ICC 3325 & 0.979 & ICC 9848 and ICC 6279 & 0.958 \\
\hline ICC 13599 and ICC 3325 & 0.979 & ICC 11764 and ICC 7184 & 0.958 \\
\hline ICC 12328 and ICC 7554 & 0.979 & ICC 14831 and ICC 13441 & 0.958 \\
\hline ICC 12328 and ICC 12155 & 0.979 & ICC 8151 and ICC 6874 & 0.957 \\
\hline \multicolumn{4}{|c|}{$\begin{array}{l}\text { Minimum: } 0.415 \text { (ICC } 12028 \text { and ICC 7819), Maximum: } 1.00 \text { (ICC13816 } \\
\text { and ICC 3325); Range genetic distance: } 0.585 \text { amongst } 465 \text { pairs }\end{array}$} \\
\hline \multicolumn{4}{|c|}{$\underline{\text { Susceptible group }}$} \\
\hline ICC 8151 and ICC 1180 & 1.000 & ICC 15697 and ICC 8384 & 0.958 \\
\hline ICC 9586 and ICC 8151 & 1.000 & ICC 7255 and ICC 1180 & 0.957 \\
\hline ICC 16207 and ICC 8151 & 1.000 & ICC 12866 and ICC 1180 & 0.957 \\
\hline ICC 15518 and ICC 9586 & 0.979 & ICC 15333 and ICC 1180 & 0.957 \\
\hline ICC 8151 and ICC 440 & 0.979 & ICC 15618 and ICC 7668 & 0.957 \\
\hline ICC 10755 and ICC 9586 & 0.979 & ICC 15333 and ICC 12307 & 0.957 \\
\hline ICC 16207 and ICC 15518 & 0.978 & ICC 10755 and ICC 440 & 0.957 \\
\hline ICC 9586 and ICC 9137 & 0.978 & ICC 16374 and ICC 10755 & 0.957 \\
\hline ICC 8384 and ICC 7668 & 0.958 & ICC 15518 and ICC 1098 & 0.938 \\
\hline ICC 8384 and ICC 8151 & 0.958 & ICC 15533 and ICC 3362 & 0.956 \\
\hline \multicolumn{4}{|c|}{$\begin{array}{l}\text { Minimum: } 0.277 \text { (Annigeri and ICC 16374); Maximum: } 1.000 \text { (ICC } 8151 \\
\text { and ICC 1180); Range genetic distance: } 0.723 \text { amongst } 276 \text { pairs }\end{array}$} \\
\hline
\end{tabular}

linear correlation between morphological and molecular similarities as recently evidenced among 19 maize inbreds (correlation ranged from 0.47 for inbred line L 217 to 0.76 for inbred L 86, average correlation over all studied inbred lines is 0.64) (Babić et al., 2012). Lack of correspondence between molecular- and phenotype-based clustering is probably due to no or a weak correlation coefficient between molecular and quantitative measures of genetic variation (Reed and Frankham, 2001; Johnson et al., 2007; Najaphy et al., 2012).

Resistance to stress imposes a cost on the fitness of plants, which means that when breeders select for one trait, such as yield, less resources remain for other functions. Such trade-offs, which may result either due to genetic linkage or pleiotropic effects, have been reported between yield and stress (pathogen or herbivore or herbicide) resistance, yield and nutrition, or seed size and seed number (Brown, 2002; Burdon and Thrall, 2003; Morris and Sands, 2006; Sadras, 2007; Vila-Aiub et al., 2009). However, deviation to this widely accepted generic view has also been reported in the literature. For example, reports in barley indicate a cost associated with the mlo gene for resistance to powdery mildew (Blumeria graminis) (Schwarzbach, 1976; Bjørnstad and Aastveit, 1990), while others detect no cost associated with resistance to barley powdery mildew (Kølster et al., 1986; Kølster and Stølen, 1987). In the present study, mean grain yield across the seven environments, for the abiotic resistant $\left(1293 \mathrm{~kg} \mathrm{ha}^{-1}\right)$ and biotic resistant $\left(1182 \mathrm{~kg} \mathrm{ha}^{-1}\right)$ accessions and susceptible $\left(1307 \mathrm{~kg} \mathrm{ha}^{-1}\right)$ accessions, differed significantly $(P-0.05)$, while those in abiotic stress resistant (1293 $\mathrm{kg} \mathrm{ha}^{-1}$ ) accessions was comparable to that of the susceptible group $\left(1307 \mathrm{~kg} \mathrm{ha}^{-1}\right)$. The biotic stress resistant accessions flowered 5 to $7 \mathrm{~d}$ later than the other groups, which flowered in 56 to $58 \mathrm{~d}$. Such differences were also noticed in per day productivity; the biotic stress resistant accessions had lower mean productivity $\left(18.8 \mathrm{~kg} \mathrm{ha}^{-1}\right)$ than the other groups $\left(22.5-23.1 \mathrm{~kg} \mathrm{ha}^{-1}\right)$. The challenge is to minimize any possible negative trade-offs between yields (or yield components) and stress resistance. Trade-offs arising from linkage could be easily overcome through recombinant breeding coupled with rigorous selection for desired traits using applied genomic tools (Brown, 2002, and references therein). Near-isogenic lines (NILs) are the ideal genetic resource to study the trade-off between resistance gene(s) and yield and yield attributing traits. The breeder's best approach in the situation of the negative trade-off would be to select moderate resistance along with good agronomic traits. An incremental increase in level of resistance might be the best approach to address negative trade-offs in crop breeding. Breeding efforts at ICRISAT and elsewhere have been successful in combining disease resistance into improved genetic backgrounds with high yield potential and specific adaptation. For example, short duration and resistance to fusarium wilt and/or heat tolerance has been successfully combined into newly developed Kabuli (ICCV 2) and desi (ICCV 88202) cultivars, which has extended the chickpea cultivation in tropical environments in India, Myanmar, Sudan, and Tanzania (ICRISAT, 2012).

This study detected a number of germplasm lines that were agronomically comparable or superior to controls, have resistance to multiple stresses, and have specific adaptations to either irrigated (ICC 637, 7441, 10399, 15686), non-irrigated (ICC 4872), or both environments (ICC 3325 and ICC 14402). In the susceptible group, a number of accessions showed specific adaptation to either of the environments, for example, ICC 1098, 3362, 9586, and 
12307 to irrigated, ICC 15680 to non-irrigated, and ICC 440, 1098, 3362, 8621, and 12307 to both environments. These accessions are thus ideal resources to broaden and enhance the genetic base of chickpea allowing the crop to withstand the vagaries of global warming and sustain or increase chickpea production worldwide. These germplasm lines meet the needs of breeders and are genetically diverse with agronomically desirable characteristics for cultivar development. These accessions may also be used as founder lines to develop multi-parent advanced generation inter-cross (MAGIC) populations to map quantitative trait loci (QTL) controlling complex traits to a small confidence interval (Valdar et al., 2006a, 2006b). Such populations can also be used as source materials for the development of cultivars. Chickpea researchers worldwide can obtain limited seed samples of these accessions from ICRISAT genebank for research purposes through a Standard Materials Transfer Agreement.

\section{References}

Abbo, S., J. Berger, and N.C. Turner. 2003. Evolution of cultivated chickpea: Four bottlenecks limit diversity and constrain adaptation. Funct. Plant Biol. 30:1081-1087. doi:10.1071/FP03084

Babić, M., V. Babi, S. Prodanovi, M. Filipovi, and V. Andjelkovi. 2012. Comparison of morphological and molecular genetic distances of maize inbreds. Genetika 44:119-128. doi:10.2298/GENSR1201119B

Bjørnstad, A., and K. Aastveit. 1990. Pleiotropic effects on the $m$-lo mildew resistance gene in barley in different genetical backgrounds. Euphytica 46:217-226. doi:10.1007/BF00027221

Brown, J.K.M. 2002. Yield penalties of disease resistance in crops. Curr. Opin. Plant Biol. 5:339-344. doi:10.1016/S13695266(02)00270-4

Burdon, J.J., and P.H. Thrall. 2003. The fitness costs to plants of resistance to pathogens. Genome Biol. 4:227. doi:10.1186/ gb-2003-4-9-227

Dua, R.P., S.K. Chaturvedi, and S. Sewak. 2001. Reference varieties of chickpea for IPR regime. Indian Inst. Pulse Res., Kanpur, India. p. 34.

Dwivedi, S.L., M.W. Blair, H.D. Upadhyaya, R. Serraj, J. Balaji, H.K. Buhariwalla, et al. 2005. Using genomics to exploit grain biodiversity in crop improvement. Plant Breed. Rev. 26:171-310.

El-Swaify, S.A., P. Pathak, T.J. Rigo, and S. Singh. 1985. Soil management for optimized productivity under rainfed conditions in the semi-arid tropics. Adv. Soil Sci. 1:1-64. doi:10.1007/978-1-4612-5046-3_1

FAO. 2011. FAOSTATS. Food and Agriculture Organization of the United Nations, Rome, Italy. http://faostat.fao.org/ site/567/default.aspx\#ancor (accessed on 4 Apr. 2013).

Frankel, O.H. 1984. Genetic perspective of germplasm conservation. In: W. Arber et al., editors, Genetic manipulations: Impact on man and society. Cambridge Univ. Press, Cambridge, UK. p. 161-470.

Gaur, P.M., S. Tripathi, C.L.L. Gowda, G.V. Ranga Rao, H.S. Sharma, S. Pande, et al. 2010. Chickpea seed production manual. Int. Crops Research Institute for the Semi-Arid Tropics, Patancheru 502 324, Andhra Pradesh, India. p. 28.

ICRISAT. 2009. ICRISAT archival report 2009. International Crops Research Institute for the Semi-Arid Tropics,
Patancheru 502 324, Andhra Pradesh, India. http://intranet/ ddg/Admin\%20Pages2009/Archival_Report_2009.aspx (accessed 20 Mar. 2013).

ICRISAT. 2012. The jewels of ICRISAT. International Crops Research Institute for the Semi-Arid Tropics, Patancheru 502 324, Andhra Pradesh, India. p. 69.

Johnson, R.C., T.J. Kisha, and M.A. Evans. 2007. Characterizing safflower germplasm with AFLP molecular markers. Crop Sci. 47:1728-1736. doi:10.2135/cropsci2006.12.0757

Jukanti, A.K., P.M. Gaur, C.L.L. Gowda, and R.N. Chibbar. 2012. Nutritional quality and health benefits of chickpea (Cicer arietinum L.): A review. Br. J. Nutr. 108(Suppl. 1):S11S16. doi:10.1017/S0007114512000797

Kashiwagi, J., L. Krishnamurthy, S. Singh, P.M. Gaur, H.D. Upadhyaya, J.D.S. Panwar, et al. 2006a. Relationships between transpiration efficiency and carbon isotope discrimination in chickpea (Cicer arietinum L.). J SAT Agric. Res. 2:1-3.

Kashiwagi, J., L. Krishnamurthy, S. Singh, and H.D. Upadhyaya. 2006b. Variation in SPAD chlorophyll meter readings (SCMR) in the minicore germplasm of chickpea. Int. Chickpea Pigeonpea Newsl. 13:16-18.

Kashiwagi, J., L. Krishnamurthy, H.D. Upadhyaya, and P.M. Gaur. 2008. Rapid screening technique for crop canopy temperature status and its relevance to drought tolerance improvement in chickpea. J. SAT Agric. Res. 6:104-105.

Kashiwagi, J., L. Krishnamurthy, H.D. Upadhyaya, H. Krishna, S. Chandra, V. Vadez, et al. 2005. Genetic variability of drought-avoidance root traits in the minicore germplasm collection of chickpea (Cicer arietinum L.). Euphytica 146:213222. doi:10.1007/s10681-005-9007-1

Kashiwagi, J., H.D. Upadhyaya, and L. Krishnamurthy. 2010. Significance and genetic diversity of SPAD chlorophyll meter reading in chickpea germplasm in the semi-arid environments. J. Food Legumes 23:99-105.

Kølster, P., L. Munk, O. Stølen, and J. Løhde. 1986. Near-isogenic barley lines with genes for resistance to powdery mildew. Crop Sci. 26:903-907. doi:10.2135/cropsci1986.0011183X00 $2600050014 \mathrm{x}$

Kølster, P., and O. Stølen. 1987. Barley isolates with genes for resistance to Erysiphe graminis f. sp. hordei in the recurrent parent 'Siri'. Plant Breed. 98:79-82. doi:10.1111/j.1439-0523.1987.tb01096.x

Krishnamurthy, L., P.M. Gaur, P.S. Basu, S.K. Chaturvedi, S. Tripathi, V. Vadez, et al. 2011a. Large genetic variation for heat tolerance in the reference collection of chickpea (Cicer arietinum L.) germplasm. Plant Genet. Resour. 9:59-69. doi:10.1017/S1479262110000407

Krishnamurthy, L., J. Kashiwagi, P.M. Gaur, H.D. Upadhyaya, and V. Vadez. 2010. Sources of tolerance to terminal drought in the chickpea (Cicer arietinum L.) mini core germplasm. Field Crops Res. 119:322-330. doi:10.1016/j.fcr.2010.08.002

Krishnamurthy, L., N.C. Turner, P.M. Gaur, H.D. Upadhyaya, R.K. Varshney, K.H.M. Siddique, et al. 2011b. Consistent variation across soil types in salinity resistance of a diverse range of chickpea (Cicer arietinum L.) genotypes. J. Agron. Crop Sci. 197:214-227. doi:10.1111/j.1439-037X.2010.00456.x

Kumar, S., S. Gupta, S. Chandra, and B.B. Singh. 2004. How wide is the genetic base of pulse crops. In: M. Ali et al., editors, Pulses in new perspective. Army Printing Press, Lucknow, India. p. 211-221.

Kumar, J., M.P. Haware, and J.B. Smithson. 1985. Registration of four short duration fusarium wilt resistant kabuli (garbanzo) chickpea germplasm. Crop Sci. 25:576-577. doi:10.2135/crop 
sci1985.0011183X002500030047x

Marshall, D.R. 1989. Limitations to the use of germplasm collections. In: A.D.H. Brown et al., editors, The use of plant genetic resources. Univ. Press, Cambridge, UK. p. 105-120.

Morris, C.E., and D.C. Sands. 2006. The breeder's dilemma-yield or nutrition? Nat. Biotechnol. 24:1078-1080. doi:10.1038/ nbt0906-1078

Mulwa, R.M.S., P.K. Kimurto, and B.K. Towett. 2010. Evaluation and selection of drought and pod borer (Helicoverpa armigera) tolerant chickpea genotypes for introduction in semi-arid areas of Kenya. In E. Adipala et al., editors, Proceedings of the Second RUFORUM Biennial Regional Conference on Building Capacity for Food Security in Africa, 20-24 Sept. 2010. RUFORUM Working Doc. Ser. No. 5, Entebbe, Uganda.

Najaphy, A., R.A. Parchin, and E. Farshadfar. 2012. Comparison of phenotypic and molecular characterization of some important wheat cultivars and advanced breeding lines. Austr. J. Crop Sci. 6:326-332.

Nass, L.L., and E. Paterniani. 2000. Pre-breeding: A link between genetic resources and maize breeding. Sci. agric. 57:581-587.

Pande, S., G.K. Kishore, H.D. Upadhyaya, and J.N. Rao. 2006. Identification of sources of multiple disease resistance in minicore collection of chickpea. Plant Dis. 90:1214-1218. doi:10.1094/PD-90-1214

Parameshwarappa, S.G., and P.M. Salimath. 2008. Field screening of chickpea genotypes for drought resistance. Karnataka J. Agric. Sci. 21:113-114.

Perrier, X., A. Flori, and F. Bonnot. 2003. Data analysis methods. In: P. Hamon et al., editors, Genetic diversity of cultivated tropical plants. Science Publishers, Enfield, NH. p. 43-76.

Reed, D.H., and R. Frankham. 2001. How closely correlated are molecular and qualitative measures of genetic variation? A meta-analysis. Evolution 55:1095-1103.

Rupela, O.P. 1987. Nodulation and nitrogen fixation in chickpea. In: M.C. Saxena and K.B. Singh, editors, The chickpea. CAB International, Wallingford, U.K. p. 191-196.

Ryan, J.G. 1997. A global perspective on pigeonpea and chickpea sustainable production systems: Present status and future potential. In: A.N. Asthana and M. Ali, editors, Recent advances in pulses research. Indian Society of Pulses Research and Development, IIPR, Kanpur, India. p. 1-31.

Sadras, V.O. 2007. Evolutionary aspects of the trade-off between seed size and number in crops. Field Crops Res. 100:125-138. doi:10.1016/j.fcr.2006.07.004

Schwarzbach, E. 1976. The pleiotropic effects of the mlo gene and their implications in breeding. In: H. Gaul, editor, Barley genetics III. Proceedings of the 3rd International Barley Genetics Symposium, Garching, Germany. 7-12 Jul. 1975. Verlag Karl Thiemig, Müchen, Germany. p. 440-445.

Serraj, R., L. Krishnamurthy, and H.D. Upadhyaya. 2004. Screening chickpea minicore germplasm for tolerance to soil salinity. Int. Chickpea and Pigeonpea Newsl. 11:29-32.

Smithson, J.B., J.A. Thompson, and R.J. Summerfield. 1985.
Chickpea (Cicer arietinum L.). In: R.J. Summerfield and E.H. Roberts, editors, Grain legume crops, Collins Publications, London, UK. p. 312-319.

Taran, B., T.D. Warkentin, A. Vandenberg, and F.A. Holm. 2010. Variation in chickpea germplasm for tolerance to imazethapyr and imazamox herbicides. Can. J. Plant Sci. 90:139-142. doi:10.4141/CJPS09061

Upadhyaya, H.D., N. Dronavalli, C.L.L. Gowda, and S. Singh. 2011. Identification and evaluation of chickpea germplasm for tolerance to heat stress. Crop Sci. 51:2079-2094. doi:10.2135/ cropsci2011.01.0018

Upadhyaya, H.D., S.L. Dwivedi, M. Baun, R.K. Varshney, S.M. Udupa, C.L.L. Gowda, et al. 2008. Genetic structure, diversity, and allelic richness in composite collection and reference set in chickpea (Cicer arietinum L.). BMC Plant Biol. 8:106. doi:10.1186/1471-2229-8-106

Upadhyaya, H.D., B.J. Furman, S.L. Dwivedi, S.M. Udupa, C.L.L. Gowda, M. Baum, et al. 2006. Development of a composite collection for mining germplasm possessing allelic variation for beneficial traits in chickpea. Plant Genet. Resour. 4:13-19. doi:10.1079/PGR2005101

Upadhyaya, H.D., and R. Ortiz. 2001. A mini core subset for capturing diversity and promoting utilization of chickpea genetic resources in crop improvement. Theor. Appl. Genet. 102:1292-1298. doi:10.1007/s00122-001-0556-y

Vadez, V., L. Krishnamurthy, R. Serraj, P.M. Gaur, H.D. Upadhyaya, D.A. Hoisington, et al. 2007. Large variation in salinity tolerance in chickpea is explained by differences in sensitivity at the reproductive stage. Field Crops Res. 104:123-129. doi:10.1016/j.fcr.2007.05.014

Valdar, W., J. Flint, and R. Mott. 2006a. Simulating the collaborative cross: Power of quantitative trait loci detection and mapping resolution in large sets of recombinant inbred strains of mice. Genetics 172:1783-1797. doi:10.1534/genetics.104.039313

Valdar, W., L.C. Solberg, D. Gauguier, S. Burnett, P. Klenerman, W.O. Cookson, et al. 2006b. Genome-wide genetic association of complex traits in heterogenous stock mice. Nat. Genet. 38:879-887. doi:10.1038/ng1840

Vila-Aiub, M.M., P. Neve, and S.B. Powles. 2009. Fitness costs associated with evolved herbicide resistance alleles in plants. New Phytol. 184:751-767. doi:10.1111/j.1469-8137.2009.03055.x

VSN International. 2013. GenStat software for windows. Release 14.1. VSNL International Ltd., Hemel Hempstead, Hertfordshire, UK.

Zaman-Allah, M., D.M. Jenkinson, and V. Vadez. 2011a. A conservative pattern of water use, rather than deep or profuse rooting, is critical for the terminal drought tolerance of chickpeas. J. Exp. Bot. 62:4239-4252. doi:10.1093/jxb/err139

Zaman-Allah, M., D.M. Jenkinson, and V. Vadez. 2011b. Chickpea genotypes contrasting for seed yield under terminal drought stress in the field differ for traits related to the control of water use. Funct. Plant Biol. 38:270-281. doi:10.1071/FP10244 